\title{
REVIEW
}

\section{Reducing work related psychological ill health and sickness absence: a systematic literature review}

\section{S Michie, S Williams}

Occup Environ Med 2003;60:3-9

A literature review revealed the following: key work factors associated with psychological ill health and sickness absence in staff were long hours worked, work overload and pressure, and the effects of these on personal lives; lack of control over work; lack of participation in decision making; poor social support; and unclear management and work role. There was some evidence that sickness absence was associated with poor management style. Successful interventions that improved psychological health and levels of sickness absence used training and organisational approaches to increase participation in decision making and problem solving, increase support and feedback, and improve communication. It is concluded that many of the work related variables associated with high levels of psychological ill health are potentially amenable to change. This is shown in intervention studies that have successfully improved psychological health and reduced sickness absence.

See end of article for authors' affiliations

Dr S Michie, Reader in Clinical Health Psychology Centre for Outcomes

Research and Effectiveness, Department of Psychology, University College London,

Gower Street, London

WCIE 6BT, UK;

s.michie@ucl.ac.uk

Accepted 14 May 2002 evels of ill health, both physical and psychological, and associated sickness absence are - high among those working in health care in the UK. ${ }^{12}$ This problem is not unique to the UK. ${ }^{3}$ Poor psychological health and sickness absence are likely to lead to problems for patients in that both the quantity and quality of patient care may be diminished. Because most health care is provided by staff working in teams, ill health and sickness absence in any one individual is likely to cause increased work and stress for other staff.

Several explanations have been put forward for this high level of ill health, including the nature of the work, organisational changes, and the large amounts and pressure of work. ${ }^{4}$ A comparison across UK hospitals in the public sector found $17 \%$ to $33 \%$, with lower rates in hospitals characterised by smaller size, greater cooperation, better communication, more performance monitoring, a stronger emphasis on training, and allowing staff more control and flexibility in their work. ${ }^{5}$ This supports the notion that organisational factors may contribute to the level of psychological ill health experienced by staff.

To tackle the problem of work related psychological ill health, evidence is needed about the work factors associated with psychological ill health and sickness absence, and about interventions that have been implemented successfully to that rates of psychological ill health varied from prevent or reduce psychological ill health and sickness absence. The primary focus of this review is the association between work factors and psychological ill health among health care staff. However, because of the paucity of evidence in health care, ${ }^{1}$ evidence was reviewed across all work settings, although presented separately for health care workers where appropriate.

\section{METHODS}

Our review method was based on that used by the NHS Centre for Reviews and Dissemination. ${ }^{6}$ This method involves a systematic examination of selected databases using a variety of strategies, including keywords and subject headings. It allows the integration of quantitative data across studies, where they have similar outcome measures, and the summary of findings where methods used are diverse.

\section{Identification of papers}

Four electronic databases were used: Medline (1987-99), PsychInfo (1987-99), Embase (199199), and the Cochrane Controlled Trials Register (1987-99). Relevant papers up to and including 1997 were selected from a larger study. The search strategy in the larger study was of MeSH key words and text words in each of three categories: work factors; staff; and ill health/ absenteeism/economic consequences. The search included all types of employment and all developed countries but was limited to abstracts in English. Secondary references were chosen from the primary paper references and by contacting academics researching this area. Psychological ill health included measures of anxiety, depression, emotional exhaustion, and psychological distress ("stress" was excluded since it is a mediating hypothetical construct rather than an outcome measure of psychological ill health). For the purpose of this review, papers from 1998 and 1999 were identified using the same search strategy, but excluding physical ill health and economic consequences.

\section{Selection criteria}

Abstracts were selected for retrieval of the paper if they were judged to include data about both work factors and psychological ill health or absenteeism. Dissertations were excluded, as were studies of very specific staff groups or settings, work patterns (for example, shift working), or events (for example, violence). All abstracts were selected independently by two researchers (three researchers were involved in this activity). The percentage of abstracts for which two researchers agreed about inclusion and exclusion varied 
Table 1 Summary of observational studies of associations between work factors and ill health: health care workers in UK

\begin{tabular}{|c|c|c|c|c|c|c|}
\hline Study & Design & Participants & $\begin{array}{l}\text { Response } \\
\text { rate }\end{array}$ & Work factors & Outcomes & Results \\
\hline $\begin{array}{l}\text { Agius et al, } \\
1996^{7}\end{array}$ & Cross sectional & $\begin{array}{l}375 \text { consultant } \\
\text { doctors }\end{array}$ & $75 \%$ & Work demands & $\begin{array}{l}\text { Emotional exhaustion } \\
\text { (MBI) }\end{array}$ & $\begin{array}{l}\text { High academic work demands associated } \\
\text { with low emotional exhaustion }(r=-0.14 \text {, } \\
p<0.05)\end{array}$ \\
\hline $\begin{array}{l}\text { Baglioni et al, } \\
1990^{8}\end{array}$ & Cross sectional & 475 senior nurses & $80 \%$ & Workload & Mental health (CCEI) & No association \\
\hline $\begin{array}{l}\text { Baldwin et al, } \\
1997^{\circ}\end{array}$ & Longitudinal & 142 junior doctors & $95 \%$ & Long hours & $\begin{array}{l}\text { Psychological distress } \\
(G H Q-28)\end{array}$ & $\begin{array}{l}\text { No association overall; association with } \\
\text { somatic symptoms, } r=0.24\end{array}$ \\
\hline $\begin{array}{l}\text { Cooper et al, } \\
1988^{10}\end{array}$ & Cross sectional & 484 dentists & $85 \%$ & $\begin{array}{l}\text { Time pressures, } \\
\text { pay stressors and } \\
\text { technical problems }\end{array}$ & Mental health (CCEI) & $\begin{array}{l}\text { Time pressures, pay stressors and technical } \\
\text { problems associated with poor mental } \\
\text { health (respective } B s=0.24,0.20,0.12 \\
F=20.54, p<0.001 \text { ) }\end{array}$ \\
\hline $\begin{array}{l}\text { Deary et al, } \\
1996^{11}\end{array}$ & Cross sectional & $\begin{array}{l}333 \text { consultant } \\
\text { doctors }\end{array}$ & $67 \%$ & Clinical workload & $\begin{array}{l}\text { Psychological distress } \\
\text { (GHQ-28), emotional } \\
\text { exhaustion (MBI) }\end{array}$ & $\begin{array}{l}\text { High clinical workload associated with } \\
\text { emotional exhaustion }\left(\chi^{2} \text { for model }=30.31 \text {, }\right. \\
p=0.11 \text {, satisfactory fit })\end{array}$ \\
\hline $\begin{array}{l}\text { Heyworth et al, } \\
1993^{12}\end{array}$ & Cross sectional & $\begin{array}{l}201 \text { trainee and } \\
\text { consultant doctors }\end{array}$ & $72 \%$ & $\begin{array}{l}\text { Task clarity, } \\
\text { supportive } \\
\text { communication }\end{array}$ & Depression (CES-D) & $\begin{array}{l}\text { Task clarity and supportive communication } \\
\text { associated with lower depression }(r=-0.51 \\
\text { and }-0.36 \text { respectively, } p=0.0001)\end{array}$ \\
\hline Parkes, $1982^{13}$ & Experimental & 164 student nurses & $97 \%$ & $\begin{array}{l}\text { Job demand, } \\
\text { discretion, social } \\
\text { support }\end{array}$ & $\begin{array}{l}\text { Anxiety (GHQ), } \\
\text { depression (CCEI), } \\
\text { sickness absence }\end{array}$ & $\begin{array}{l}\text { Anxiety and depression associated with low } \\
\text { job discretion and job support ( } r=-0.30 \\
\text { and }-0.35 \text { for anxiety and }-0.26 \text { and } \\
-0.36 \text { for depression) and job demand was } \\
\text { negatively associated with sickness absence } \\
(r=-0.24)\end{array}$ \\
\hline Quine, $1999^{14}$ & Cross sectional & $\begin{array}{l}1100 \text { health care } \\
\text { workers }\end{array}$ & $70 \%$ & Bullying & $\begin{array}{l}\text { Anxiety, depression } \\
\text { (HADS) }\end{array}$ & $\begin{array}{l}\text { Bullying associated with higher anxiety } \\
(30 \% \text { v } 9 \%, p<0.001) \text { and depression }(8 \% \\
\text { v } 1 \%, p<0.001)\end{array}$ \\
\hline $\begin{array}{l}\text { Rees and Cooper, } \\
1992^{15}\end{array}$ & Cross sectional & $\begin{array}{l}1176 \text { health care } \\
\text { workers }\end{array}$ & $67 \%$ & $\begin{array}{l}\text { Control over work } \\
\text { (OSI) }\end{array}$ & Sickness absence & No association \\
\hline $\begin{array}{l}\text { Sutherland and } \\
\text { Cooper, } 1993^{16}\end{array}$ & Cross sectional & 917 family doctors & $61 \%$ & Job demands & $\begin{array}{l}\text { Anxiety, depression } \\
\text { and somatic anxiety } \\
\text { (CCEI) }\end{array}$ & $\begin{array}{l}\text { Associations with anxiety (beta }=0.17 \text { ) } \\
\text { depression }(b e t a=0.28) \text { and somatic } \\
\text { anxiety (beta }=0.23 \text { ) }\end{array}$ \\
\hline $\begin{array}{l}\text { Tyler and } \\
\text { Cushway, } 1992^{17}\end{array}$ & Cross sectional & 72 nurses & $60 \%$ & $\begin{array}{l}\text { Workload, conflict, } \\
\text { social support }\end{array}$ & $\begin{array}{l}\text { Psychological distress } \\
\text { (GHQ 28) }\end{array}$ & $\begin{array}{l}\text { GHQ predicted by managing the workload } \\
\text { (beta }=0.32 \text { ) }\end{array}$ \\
\hline
\end{tabular}

between $80 \%$ and $90 \%$. Disagreements were resolved by discussion

\section{Information extraction}

Information from papers was extracted and coded within the following categories: study aim, study design, type of study population (for example, occupational group), sampling strategy, sample size and response rate, demographic characteristics, type of intervention, type of study measure, main outcomes, and summary of results.

\section{Further selection criteria}

Coded papers excluded from the review were studies with: volunteer or inadequately described sample; response rate of less than 60\%; no standardised measures of psychological outcome.

\section{RESULTS}

Of the studies identified as part of the larger study, ${ }^{1} 40$ were selected for this study ( 34 associations and six interventions). A further nine studies meeting the above selection criteria were identified in the period 1998-99, all of associations. No studies were found in the Cochrane Controlled Trials Register. The results are summarised in tables $1-4$.

Because these studies were diverse in terms of outcomes and measures used to assess these outcomes, a meta-analysis was not appropriate.

\section{Associations with work}

The results are presented in three groups: health care workers in the UK, health care workers in other developed countries, and non-health care workers. This enabled an assessment of whether associations between work factors and psychological ill health are similar across sector and country.

\section{Health care}

In the UK, factors associated with psychological ill health in doctors, from junior to senior grades, are long hours worked, ${ }^{9}$ high workload and pressure of work, ${ }^{71611}$ and lack of role clarity $^{12}$ (table 1). Pressure of work has also been found to be associated with poor mental health in dentists. ${ }^{10}$ In family doctors, the issues were interruptions during and outside surgery hours and patient demands. ${ }^{16}$

Among UK nurses, the most frequently reported source of psychological ill health was workload pressures. ${ }^{17}$ Distress in student nurses has been caused by low involvement in decision making and use of skills, and low social support at work. ${ }^{13}$ In a study of health care workers across job type, bullying was found to be prevalent, carried out mainly by managers and associated with both anxiety and depression. ${ }^{14}$ Of the two studies addressing sickness absence, one found a negative association with job demands, ${ }^{13}$ while the other found no association with control over work. ${ }^{15}$

Similar factors are associated with psychological ill health in health care workers in the rest of Europe, the USA, and Australia (table 2). The one study of doctors found an association between work control and social support and psychological distress. ${ }^{22}$ Among nurses, lack of co-worker support, ${ }^{24}{ }^{27}$ job influence, ${ }^{26}$ and organisational climate and role ambiguity ${ }^{28}$ were associated with psychological distress. Among other hospital workers, work overload and pressure, role ambiguity, lack of control over work, and lack of participation in decision making were all found to be associated with distress. ${ }^{182025}$

Sickness absence was associated with work pressures and lack of training, ${ }^{23}$ unsupportive management style, ${ }^{21}$ role ambiguity, tolerance of absenteeism, and low pay. ${ }^{19}$

\section{Beyond health care}

The picture among non-health care workers in Europe and the USA was similar to that of health care workers (table 3 ). The 
Table 2 Summary of observational studies of associations between work factors and ill health: health care workers in developed countries beyond the UK

\begin{tabular}{|c|c|c|c|c|c|c|c|}
\hline Study & Country & Design & Participants & $\begin{array}{l}\text { Response } \\
\text { rate }\end{array}$ & Work factors & Outcomes & Results \\
\hline $\begin{array}{l}\text { Arsenault et al, } \\
1991^{18}\end{array}$ & - Canada & Cross sectional & $\begin{array}{l}760 \text { hospital } \\
\text { workers }\end{array}$ & $\begin{array}{l}\text { Not } \\
\text { reported }\end{array}$ & $\begin{array}{l}\text { Professional latitude, } \\
\text { clinical demands, workload } \\
\text { problems, role difficulties }\end{array}$ & $\begin{array}{l}\text { Mental strain } \\
\text { including depression } \\
\text { (Cobb) and anxiety } \\
\text { (STAI) }\end{array}$ & $\begin{array}{l}\text { Low professional latitude }(F=12.7 \text {, } \\
\mathrm{p}<0.001) \text { and high workload } \\
\text { problems }(\mathrm{F}=4.5, \mathrm{p}<0.04) \text { and } \\
\text { role difficulties }(\mathrm{F}=31.6, \mathrm{p}<0.001) \\
\text { associated with mental strain }\end{array}$ \\
\hline $\begin{array}{l}\text { Brooke and } \\
\text { Price, } 1989^{19}\end{array}$ & USA & Cross sectional & $\begin{array}{l}425 \text { hospital } \\
\text { workers }\end{array}$ & $74 \%$ & $\begin{array}{l}\text { Routinisation, } \\
\text { centralisation, pay, reward } \\
\text { policy, role ambiguity, } \\
\text { conflict, overload, } \\
\text { organisational tolerance of } \\
\text { absenteeism }\end{array}$ & Absenteeism & $\begin{array}{l}\text { High role ambiguity and tolerance } \\
\text { of absenteeism, low pay and low } \\
\text { centralisation predicted } \\
\text { absenteeism (structural coefficients } \\
0.21, p<0.001 ; 0.27, p<0.001 \text {; } \\
-0.11, p<0.05 ;-0.19, p<0.02 \text {, } \\
\text { respectively) }\end{array}$ \\
\hline $\begin{array}{l}\text { Estryn-Behar et } \\
\text { al, } 1990^{20}\end{array}$ & France & Cross sectional & $\begin{array}{l}1505 \text { female } \\
\text { hospital } \\
\text { workers }\end{array}$ & $90 \%$ & $\begin{array}{l}\text { Mental load, insufficient } \\
\text { training, time pressure }\end{array}$ & $\begin{array}{l}\text { Psychological } \\
\text { distress (GHQ-12) }\end{array}$ & $\begin{array}{l}\text { Mental load and time pressure } \\
\text { associated with psychological } \\
\text { distress (ORs } 2.9 \text { and } 2.2 \text { ) }\end{array}$ \\
\hline $\begin{array}{l}\text { Gray-Toft and } \\
\text { Anderson, } \\
1985^{21}\end{array}$ & USA & Experimental & 159 nurses & $\begin{array}{l}\text { Not } \\
\text { reported }\end{array}$ & $\begin{array}{l}\text { Open, supportive } \\
\text { supervisory style }\end{array}$ & Absenteeism & $\begin{array}{l}\text { Open supportive supervisory style } \\
\text { associated with lower absenteeism } \\
\text { (relevant statistics not presented) }\end{array}$ \\
\hline $\begin{array}{l}\text { Johnson et al, } \\
1995^{22}\end{array}$ & USA & Longitudinal & 581 doctors & $86 \%$ & $\begin{array}{l}\text { Job demands, work control, } \\
\text { social support }\end{array}$ & $\begin{array}{l}\text { Psychological } \\
\text { distress (GHQ-20) }\end{array}$ & $\begin{array}{l}\text { Work control and social support } \\
\text { negatively associated with } \\
\text { psychological distress }(B=-0.44 \\
p=0.05 \text { and } B=-0.46, p=0.05)\end{array}$ \\
\hline $\begin{array}{l}\text { Landeweerd } \\
\text { and Boumans, } \\
1994^{23}\end{array}$ & \multicolumn{2}{|c|}{ Netherlandsross sectional } & 561 nurses & $96 \%$ & $\begin{array}{l}\text { Work pressure, job } \\
\text { complexity, feedback, } \\
\text { autonomy, } \\
\text { promotion/training }\end{array}$ & Absence frequency & $\begin{array}{l}\text { Work pressures associated with } \\
\text { absence frequency }(B=0.12) \text { and } \\
\text { promotion/training negatively } \\
\text { associated }(B=-0.12)\end{array}$ \\
\hline $\begin{array}{l}\text { Marshall \& } \\
\text { Barnett, } \\
1992^{24}\end{array}$ & USA & Cross sectional & $\begin{array}{l}362 \text { female } \\
\text { nurses and } \\
\text { social } \\
\text { workers }\end{array}$ & $\begin{array}{l}\text { Not } \\
\text { reported }\end{array}$ & $\begin{array}{l}\text { Work related support, job } \\
\text { overload }\end{array}$ & $\begin{array}{l}\text { Psychological } \\
\text { distress (SCL-90-R) } \\
\text { and emotional } \\
\text { well-being (Rand } \\
\text { Corporation) }\end{array}$ & $\begin{array}{l}\text { Co-worker support associated with } \\
\text { emotional wellbeing }(B=-0.20 \text {, } \\
p<0.01)\end{array}$ \\
\hline $\begin{array}{l}\text { Martin, } \\
1984^{25}\end{array}$ & USA & Cross sectional & $\begin{array}{l}95 \text { and } 140 \\
\text { hospital } \\
\text { workers }\end{array}$ & $\begin{array}{l}63 \% \text { and } \\
70 \%\end{array}$ & $\begin{array}{l}\text { Work overload and } \\
\text { ambiguity, participation in } \\
\text { decision making }\end{array}$ & $\begin{array}{l}\text { Psychological } \\
\text { distress (GHQ-12) }\end{array}$ & $\begin{array}{l}\text { Work factors associated with } \\
\text { distress (canonical } \\
\text { correlations }=0.53 \text { and } 0.41 \text {, } \\
p<0.001 \text { ) }\end{array}$ \\
\hline $\begin{array}{l}\text { Petterson et al, } \\
1995^{26}\end{array}$ & Sweden & Cross sectional & 2568 nurses & $76 \%$ & Job influence & $\begin{array}{l}\text { Emotional } \\
\text { exhaustion (MBI) }\end{array}$ & $\begin{array}{l}\text { Job influence negatively associatec } \\
\text { with emotional exhaustion } \\
\text { ( } p<0.001)\end{array}$ \\
\hline $\begin{array}{l}\text { Pisarski et al, } \\
1998^{27}\end{array}$ & Australia & Cross sectional & $\begin{array}{l}172 \text { nurses, } \\
\text { aged } 21-40 \\
\text { years }\end{array}$ & $\begin{array}{l}\text { Not } \\
\text { reported }\end{array}$ & Social support & $\begin{array}{l}\text { Psychological } \\
\text { distress (GHQ-12) }\end{array}$ & $\begin{array}{l}\text { Co-worker social support directly } \\
\text { associated with distress and } \\
\text { mediates association with } \\
\text { supervisor social support (path } \\
\text { coefficients }<0.001 \text { ) }\end{array}$ \\
\hline $\begin{array}{l}\text { Revicki and } \\
\text { May, } 1989^{28}\end{array}$ & USA & Cross sectional & 232 nurses & $77 \%$ & $\begin{array}{l}\text { Organisational climate, } \\
\text { supervisor behaviour, role } \\
\text { ambiguity, social support }\end{array}$ & $\begin{array}{l}\text { Depression (Rand } \\
\text { corporation) }\end{array}$ & $\begin{array}{l}\text { The association of organisational } \\
\text { climate and role ambiguity with } \\
\text { depression is mediated by stress }\end{array}$ \\
\hline
\end{tabular}

key work factors associated with psychological ill health were:

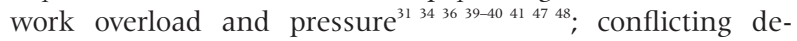
mands ${ }^{47}$; lack of control over work and lack of participation in decision making $3436394046-48$; poor social support at work 3133353839414748 ; unclear management and work role $^{29} 30413438$; interpersonal conflict ${ }^{42} 46$; and conflict between work and family demands. ${ }^{46}$ Long hours were found to be associated with depression in women, but not in men. ${ }^{44}$

Sickness absence was negatively associated with high job demand, ${ }^{45}$ and positively associated with monotonous work, not learning new skills and low control over work, ${ }^{36} 3745$ and non-participation at work. ${ }^{43}$

\section{Evaluated interventions}

Six intervention studies met our methodological criteria (table 4). Three were randomised controlled trials, ${ }^{49} 5052$ three were conducted in the USA, ${ }^{505154}$ one in the UK, ${ }^{53}$ and two in Scandinavia. ${ }^{49} 52$ Three were of health care workers. ${ }^{50} 5254$ Five were training programmes offered as part of the working day and one was an organisational intervention. ${ }^{53}$

Skills to mobilise support at work and to participate in problem solving and decision making were taught to care staff of people with mental ill health or mental disability in a randomised controlled trial. ${ }^{50}$ Groups of 20 had six sessions of 4-5 hours training over two months, and were trained to train those in their workplace. Compared to those in the control group, the intervention group reported more supportive feedback, more ability to cope, and better work team functioning and climate. Among those most at risk of leaving, those undergoing the training reported reduced depression. The second randomised controlled trial compared receiving support, advice, and feedback from a psychologist with having the passive presence of the same psychologist at staff meetings in a geriatric hospital facing organisational change. ${ }^{52}$ Staff were taught skills of stress management, and how to participate in, and control, their work. The intervention was an hour a fortnight during the 10 weeks before, and the 10 weeks after, the organisational change. There was a significant difference between groups, with a decrease of stress hormone levels in the intervention group.

Staff of a psychiatric hospital were taught verbal and nonverbal communication and empathy skills. ${ }^{54}$ Groups of 6-8 had eight hour weekly sessions for four weeks involving information, videos, modelling, and role playing. Compared to a matched control group, the intervention group showed reduced staff resignations and sick leave, although no statistical tests are reported.

Among physically inactive employees of an insurance company, a randomised controlled trial found stress management training and aerobic exercise interventions had mixed 
Table 3 Summary of observational studies of associations between work factors and ill health: non-health care workers

\begin{tabular}{|c|c|c|c|c|c|c|c|}
\hline Study & Country & Design & Participants & Response rate & Work factors & Outcomes & Results \\
\hline Bacharach ef al, $1991^{29}$ & USA & Cross sectional & $\begin{array}{l}430 \text { public sector } \\
\text { engineers }\end{array}$ & $79 \%$ & Role conflict, time pressure & Emotional exhaustion (MBI) & High role conflict $(B=0.24)$ associated with emotional exhaustion \\
\hline Carayon et al, $1995^{30}$ & USA & Prospective & $\begin{array}{l}148 \text { public sector } \\
\text { office workers }\end{array}$ & $71 \%$ & $\begin{array}{l}\text { Job demands, content and control, social } \\
\text { support, task clarity and job future } \\
\text { ambiguity }\end{array}$ & $\begin{array}{l}\text { Worker strain, including } \\
\text { anxiety and depression } \\
\text { (McNair) }\end{array}$ & $\begin{array}{l}\text { Predictors of worker strain at one and two years were task clarity } \\
\text { and job future ambiguity }\end{array}$ \\
\hline Driscoll et al, $1995^{31}$ & USA & Cross sectional & $\begin{array}{l}4900 \text { public sector } \\
\text { workers }\end{array}$ & $70 \%$ & Job demands, control and social support & $\begin{array}{l}\text { Anxiety and depression } \\
\text { (NIOSH Generic Job Stress } \\
\text { Questionnaire) }\end{array}$ & $\begin{array}{l}\text { High demand and low support associated with anxiety (ORs } 1.46 \\
\text { and 2.01) and depression (ORs } 2.02 \text { and } 1.69 \text { ) }\end{array}$ \\
\hline Ferrie et al, $1998^{32}$ & UK & Prospective & $\begin{array}{l}10308 \text { public sector } \\
\text { workers }\end{array}$ & $80 \%$ & Job insecurity & $\begin{array}{l}\text { Psychological distress } \\
(G H Q-30)\end{array}$ & Non-significant association \\
\hline Frese, $1999^{33}$ & Germany & Longitudinal & $\begin{array}{l}90 \text { male blue collar } \\
\text { workers }\end{array}$ & $35-66 \%$ & $\begin{array}{l}\text { Work intensity, uncertainty, } \\
\text { organisational problems, environmental } \\
\text { problems, social pressure, social support }\end{array}$ & Anxiety, depression & $\begin{array}{l}\text { Social pressure and support associated with anxiety/depression } \\
(r=0.21 / 0.20 \text { and }-0.21 /-0.27)\end{array}$ \\
\hline Frone et al, $1995^{34}$ & USA & Longitudinal & $\begin{array}{l}795 \text { employed } \\
\text { adults }\end{array}$ & $67 \%$ & $\begin{array}{l}\text { Work pressure, lack of autonomy, role } \\
\text { ambiguity }\end{array}$ & Depression (CES-D) & $\begin{array}{l}\text { Work pressure, lack of autonomy, role ambiguity all associated } \\
\text { with depression }(B s=0.10, p<0.61 ; 0.19, p<0.001 ; 0.18 \text {, } \\
p<0.001 \text { respectively) }\end{array}$ \\
\hline Fusilier et al, $1987^{35}$ & USA & Cross sectional & $\begin{array}{l}312 \text { police officers } \\
\text { and fire fighters }\end{array}$ & $65 \%$ & $\begin{array}{l}\text { Role conflict and ambiguity, social } \\
\text { support }\end{array}$ & Depression (Caplan) & Low social support associated with depression $(B=-0.24, p<0.01)$ \\
\hline 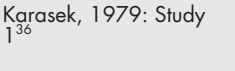 & Sweden & Longitudinal & 1896 working males & $92 \%$ and $85 \%$ & Decision latitude and job demands & $\begin{array}{l}\text { Depression (amended from } \\
\text { American Health Survey), } \\
\text { absenteeism }\end{array}$ & $\begin{array}{l}\text { Decision latitude negatively associated with depression and } \\
\text { absenteeism }(O R=-1.29, p<0.05 \text { and }-1.44, p<0.01) \text {. Job } \\
\text { demands associated with depression }(O R=1.45, p<0.001)\end{array}$ \\
\hline Study 2 & USA & Cross sectional & 911 working males & $76 \%$ & As above & As above & $\begin{array}{l}\text { Decision latitude negatively associated with depression and } \\
\text { absenteeism }(O R=-1,41, p<0.01 \text { and }-2.04,0<0.001) ; \text { i iob } \\
\text { demands associated with depression }(O R=1.20, p<0.05)\end{array}$ \\
\hline Karasek, $1990^{37}$ & Sweden & Cross sectional & $\begin{array}{l}8504 \text { white collar } \\
\text { workers }\end{array}$ & $87 \%$ & Changes in control over work & Depression and absenteeism & $\begin{array}{l}\text { Decreased control associated with depression }(p<0.01) \text { and with } \\
\text { absenteeism in men }(p<0.01) \text { but not women }\end{array}$ \\
\hline LaRocco et al, $1980^{38}$ & USA & Cross sectional & 636 male workers & Not reported & $\begin{array}{l}\text { Supervisor support, participation, future } \\
\text { ambiguity, under-utilisation, workload, } \\
\text { role conflict }\end{array}$ & $\begin{array}{l}\text { Anxiety and depression (Cobb } \\
\text { and Kasl) }\end{array}$ & $\begin{array}{l}\text { Supervisor support buffers the adverse effect of low participation } \\
\text { on depression (p }<0.1 \text {, significant }) \text { and of future ambiguity on } \\
\text { anxiety }(p<0.01)\end{array}$ \\
\hline $\begin{array}{l}\text { Niedhammer et al, } \\
1998^{39}\end{array}$ & France & Prospective & 11552 & $92 \%$ & $\begin{array}{l}\text { Psychological demands, decision latitude } \\
\text { and social support }\end{array}$ & Depression (CES-D) & $\begin{array}{l}\text { High psychological demand, low decision latitude and social } \\
\text { support associated with subsequent depression. For men, OR of } \\
1.8,1.4 \text { and } 1.6 \text { respectively and for women, OR of } 1.4,1.4 \text { and } \\
1.3 \text { respectively. }\end{array}$ \\
\hline $\begin{array}{l}\text { Payne and Fletcher, } \\
1983^{40}\end{array}$ & UK & Cross sectional & 148 teachers & $74 \%$ & Workload demands, discretion & Anxiety and depression (CCEI) & $\begin{array}{l}\text { Association with workload demands (betas }=0.117 \text { for anxiety and } \\
0.176 \text { for depression) and negative association with discretion } \\
\text { (betas }=-0.222 \text { for anxiety and }-0.121 \text { for depression) }\end{array}$ \\
\hline Reifman et al, 1991 ${ }^{41}$ & USA & $\begin{array}{l}\text { Cross sectional and } \\
\text { longitudinal }\end{array}$ & $\begin{array}{l}200 \text { married, } \\
\text { professional women }\end{array}$ & $>90 \%$ & $\begin{array}{l}\text { Social support at work, control over } \\
\text { work, role ambiguity, workload }\end{array}$ & Depression (SCL-90) & $\begin{array}{l}\text { Cross sectionally, association with social support at work }(r=0.37) \text {, } \\
\text { role ambiguity }(r=0.35) \text { and workload }(r=0.29) \text {. No associations } \\
\text { one year later }\end{array}$ \\
\hline Romanov et al, $1996^{42}$ & Finland & Prospective & 15530 employees & Not reported & Conflict at work & $\begin{array}{l}\text { Psychiatric morbidity (hospital } \\
\text { discharge registry) }\end{array}$ & Positive association (RR $\left.2.18, \mathrm{Cl}_{95} 1.34,3.54\right)$ \\
\hline $\begin{array}{l}\text { Rubenowitz et al, } \\
1983^{43} \text { is }\end{array}$ & Sweden & Cross sectional & $\begin{array}{l}25 \text { departments of } 5 \\
\text { companies. } \\
\text { Numbers not } \\
\text { reported }\end{array}$ & $85-90 \%$ & $\begin{array}{l}\text { Perceived participation (individual, group, } \\
\text { representative) }\end{array}$ & Absenteeism & Negative association for individual participation $(r=-0.53)$ \\
\hline Shields, $1999^{44}$ & Canada & Prospective & $\begin{array}{l}3830 \text { working } \\
\text { population }\end{array}$ & $80 \%$ & Long working hours & $\begin{array}{l}\text { Depression (Composite } \\
\text { International Diagnostic } \\
\text { Interview) }\end{array}$ & $\begin{array}{l}>35 \text { hours per week associated with depression in women } \\
(\mathrm{OR}=2.2) \text { but not men }\end{array}$ \\
\hline $\begin{array}{l}\text { Smulders and Nijuis, } \\
1999^{45}\end{array}$ & The Netherlands & $\begin{array}{l}\text { Cross sectional and } \\
\text { prospective }\end{array}$ & $\begin{array}{l}1755 \text { male public } \\
\text { sector workers }\end{array}$ & $70 \%$ & Job control and job demands & $\begin{array}{l}\text { Absence rate and absence } \\
\text { frequency }\end{array}$ & $\begin{array}{l}\text { Cross sectionally, iob control associated with low absence } \\
\text { frequency (beta, } 0.10, p<0.01) \text { and job demand associated with } \\
\text { low absence rate (beta }=-0.08, p<0.05 \text { ) }\end{array}$ \\
\hline $\begin{array}{l}\text { Sparks and Cooper, } \\
1999^{46}\end{array}$ & UK & Cross sectional & $\begin{array}{l}7099 \text { from } 13 \\
\text { occupations }\end{array}$ & Not reported & $\begin{array}{l}\text { Work control, career achievement, } \\
\text { organisational climate, job factors, } \\
\text { home/work interface, work relationships }\end{array}$ & Mental health (OSI) & All associated $(r=0.22$ to $-0.28, p<0.001)$ \\
\hline Stansfeld et al, $1995^{47}$ & UK & Cross sectional & $\begin{array}{l}10314 \text { public sector } \\
\text { employees }\end{array}$ & $73 \%$ & $\begin{array}{l}\text { Job variety and skill use, control, social } \\
\text { support, work pace, conflicting demands }\end{array}$ & $\begin{array}{l}\text { Psychological distress } \\
\text { (GHQ-30) }\end{array}$ & All significantly associated (intertile trend $p$ values $<0.001$ ) \\
\hline Stansfeld et al, $1998^{48}$ & UK & Prospective & $\begin{array}{l}7372 \text { public sector } \\
\text { workers }\end{array}$ & $72 \%$ & $\begin{array}{l}\text { Job demands, decision latitude, social } \\
\text { support and effort-reward imbalance }\end{array}$ & $\begin{array}{l}\text { Psychological functioning } \\
\text { (Sf-36) }\end{array}$ & $\begin{array}{l}\text { Low support and effort-reward imbalance associated with poor } \\
\text { psychological functioning (OR=1.2 for men and } 1.4 \text { for women; } \\
1.8 \text { for men and } 2.3 \text { for women respectively). In men, low decision } \\
\text { latitude }(O R=1.2 \text { ) and in women, high job demand (OR=2.0) were } \\
\text { associated with poor psychological functioning }\end{array}$ \\
\hline
\end{tabular}


effects. ${ }^{49}$ After three sessions a week for 10 weeks, stress management training resulted in improved perceived coping ability but no change in physical or psychological health. Aerobic exercise resulted in improved feelings of wellbeing and decreased complaints of muscle pain.

Employees of a fire department underwent one of seven training programmes emphasising one or more aspect of stress management: physiological processes, coping with people, or interpersonal awareness processes. ${ }^{51}$ Weekly sessions for 8-10 people were run over 42 weeks. There was no control group. Compared to baseline, there were reductions in depression, anxiety, psychological strain, and emotional exhaustion immediately after the programme. There was a further reduction in psychological strain and emotional exhaustion at 9-16 months follow up.

A structural intervention for local authority staff on long term sickness absence was effective in reducing sickness absence. Referral to occupational health services was triggered after two or three months absence, rather than at six months which was the practice before the intervention. The average duration of sickness absence reduced from 40 to 25 weeks before resumption of work and from 72 to 53 weeks for those staff who left employment for medical reasons. The authors describe large financial savings but no statistical tests are reported..$^{33}$

\section{DISCUSSION}

This systematic review of a large number of studies covers a wide range of employment sectors in the developed world and summarises those studies that use rigorous methods. The studies show that, while levels of psychological ill health are higher in health care than in non-health care workers, ${ }^{5}$ the associations between work factors and psychological ill health are similar. They are also similar across continents. This suggests that a generic approach to reducing work related psychological ill health may be appropriate.

The most common work factors associated with psychological ill health were work demand (long hours, workload, and pressure), lack of control over work, and poor support from

\section{Main messages}

- Key work factors associated with psychological ill health and sickness absence in staff are long hours worked, work overload and pressure, and the effects of these on personal lives; lack of control over work; lack of participation in decision making; poor social support; and unclear management and work role.

- There is some evidence that sickness absence is associated with poor management style.

- Successful interventions that improve psychological health and levels of sickness absence use training and organisational approaches to increase participation in decision making and problem solving, increase support and feedback, and improve communication.

managers. These were also associated with sickness absence. The findings of this review, summarised in tables $1-4$, are consistent with the demand-control model of job strain. ${ }^{36}$ Interventions aimed at changing these workplace factors reduced psychological ill health.

This review highlights limitations in the research identified. The studies that have been carried out are limited in the questions addressed and in the study designs used. Since most studies are cross sectional, causal relations cannot be shown. It may be that the associations found reflect a tendency for more vulnerable people to choose work in caring roles or other types of job which are well represented in published research studies. The question of what aspects of work lead to ill health and sickness absence can only be addressed by longitudinal studies that are able to investigate the causal relations between work factors and health outcomes and by randomised controlled trials of interventions. A longitudinal study that directly addressed the nature of the relation found a causal relation between psychological stress and psychosomatic complaints. ${ }^{55}$

There are several practical implications suggested by the studies of association in this review, for both employment

\begin{tabular}{|c|c|c|c|c|c|c|}
\hline Study & Participants & Design & $\begin{array}{l}\text { Response } \\
\text { rate }\end{array}$ & Intervention & Outcomes & Results \\
\hline $\begin{array}{l}\text { Gronningsaeter et } \\
a l, 1992^{49}\end{array}$ & $\begin{array}{l}76 \text { physically } \\
\text { inactive Norwegian } \\
\text { insurance workers }\end{array}$ & Stratified RCT & $72 \%$ & $\begin{array}{l}6 \text { sessions aerobic exercise } \\
\text { per week for } 10 \text { weeks or } \\
3 \text { sessions stress } \\
\text { management training per } \\
\text { week for } 10 \text { weeks }\end{array}$ & $\begin{array}{l}\text { Anxiety (STAl) } \\
\text { and health } \\
\text { complaints }\end{array}$ & $\begin{array}{l}\text { No association of either intervention } \\
\text { with anxiety. Aerobic exercise } \\
\text { associated with reduced health } \\
\text { complaints ( } F=3.4, p=0.07 \text { compared } \\
\text { to controls, and } F=4.8, p<0.05 \\
\text { compared to stress management } \\
\text { intervention) }\end{array}$ \\
\hline $\begin{array}{l}\text { Heaney et al, } \\
1995^{50}\end{array}$ & $\begin{array}{l}1375 \text { US residential } \\
\text { care workers }\end{array}$ & Cluster RCT & $62 \%$ & $\begin{array}{l}6 \times 4 \text { hour sessions over } 9 \\
\text { weeks to teach skills to } \\
\text { enhance social support } \\
\text { and problem solving }\end{array}$ & $\begin{array}{l}\text { Depression } \\
\text { (SCL-90R) }\end{array}$ & $\begin{array}{l}\text { For those most at risk of leaving their } \\
\text { jobs, } R^{2}=0.41, p<0.01\end{array}$ \\
\hline $\begin{array}{l}\text { Kagan et al, } \\
1995^{51}\end{array}$ & $\begin{array}{l}373 \text { US fire } \\
\text { department workers }\end{array}$ & $\begin{array}{l}\text { Randomised, } \\
\text { uncontrolled }\end{array}$ & $\begin{array}{l}\text { Not } \\
\text { reported }\end{array}$ & $\begin{array}{l}42 \text { weeks of } 7 \\
\text { psycho-educational } \\
\text { programmes, } 6 \text { weeks } \\
\text { each }\end{array}$ & $\begin{array}{l}\text { Anxiety, } \\
\text { depression, } \\
\text { psychological } \\
\text { strain, emotional } \\
\text { burnout }\end{array}$ & $\begin{array}{l}\text { Compared to baseline, } \mathrm{F}=52.3,42.2 \text {, } \\
29.1,10.6 \text { respectively; } \mathrm{p}<0.001 \text { for } \\
\text { all. } \\
\text { At } 9-16 \text { month follow up, } \mathrm{F}=4.8 \\
(\mathrm{p}<0.05), 8.7(\mathrm{p}<0.01), 21.4 \\
(\mathrm{p}<0.001), 45.2(\mathrm{p}<0.001) \\
\text { respectively }\end{array}$ \\
\hline $\begin{array}{l}\text { Lokk and Arnetz, } \\
1997^{52}\end{array}$ & $\begin{array}{l}26 \text { Swedish hospital } \\
\text { ward workers }\end{array}$ & RCT & $93 \%$ & $\begin{array}{l}20 \text { weekly } 1 \text { hour stress } \\
\text { management sessions }\end{array}$ & $\begin{array}{l}\text { Stress hormone } \\
\text { (prolactin) level }\end{array}$ & $\begin{array}{l}\text { Change scores: } \\
\text { Intervention group }-0.58 \\
\text { Control group }+1.85 \\
F=7.3, p<0.01\end{array}$ \\
\hline $\begin{array}{l}\text { Malcolm et al, } \\
1993^{53}\end{array}$ & $\begin{array}{l}604 \text { UK long term } \\
\text { sick local } \\
\text { government workers }\end{array}$ & Observational & $100 \%$ & $\begin{array}{l}\text { Early referral to } \\
\text { Occupational Health }\end{array}$ & $\begin{array}{l}\text { Duration of } \\
\text { sickness absence } \\
\text { (weeks) }\end{array}$ & $\begin{array}{l}25 \text { weeks in intervention period } \\
\text { compared to } 40 \text { in control period }\end{array}$ \\
\hline $\begin{array}{l}\text { Smoot and } \\
\text { Gonzales, } 1995^{54}\end{array}$ & $\begin{array}{l}65 \text { US hospital } \\
\text { workers }\end{array}$ & $\begin{array}{l}\text { Matched } \\
\text { controlled }\end{array}$ & $90 \%$ & $\begin{array}{l}4 \text { weekly } 8 \text { hour sessions } \\
\text { of communication training }\end{array}$ & $\begin{array}{l}\text { Sick leave (hours) } \\
\text { in } 6 \text { months after } \\
\text { compared to } 6 \\
\text { months before }\end{array}$ & $\begin{array}{l}\text { \% change: }-28.2 \text { in experimental } \\
\text { group, }-6.4 \text { in control group }\end{array}$ \\
\hline
\end{tabular}




\section{Policy implications}

- Many of the work related variables associated with high levels of psychological ill health are potentially amenable to change.

- More evaluations of interventions are required, based on randomised or longitudinal research designs.

- Interventions for which evidence of effectiveness exists should be piloted and evaluated across different work settings.

practices and management style. Intervention studies, however, have focused mainly on staff training. There is a need for future studies to evaluate interventions based on employment practices and management style. This would represent primary prevention, reducing sources of psychological ill health, rather than secondary prevention, training individuals who are already experiencing work related stress, to be more robust in the face of such pressures. Only one of the intervention studies included an economic evaluation: such evaluations are important in facilitating employers to make decisions about whether or not to implement interventions.

Future research should adhere to minimum scientific standards absent in many of the studies reviewed, such as adequate design, sufficiently large samples, and valid outcome measures. Lessons that are learnt from rigorously evaluated interventions can then be applied more generally.

\section{ACKNOWLEDGEMENTS}

We are grateful to Shriti Pattani for help with literature searching and to Frédérique Cooper for help with preparing this manuscript.

\section{Authors' affiliations}

S Michie, Reader in Clinical Health Psychology, Centre for Outcomes Research and Effectiveness, Department of Psychology, University College London, Gower Street, London WC1E 6BT, UK

S Williams, Consultant in Occupational Medicine, Royal Free

Hampstead NHS Trust, London NW3 2QG, UK

\section{REFERENCES}

1 Williams S, Michie S, Pattani S. Improving the health of the NHS workforce. London: The Nuffield Trust, 1998.

2 Confederation of British Industry. Managing absence: in sickness and in health. London: CBI, 1997.

3 Whitley TW, Allison Jr EJ, Gallery ME, et al. Work related stress and depression among practicing emergency physicians: an international study. Ann Emerg Med 1994;23:1068-71.

4 Cox T, Griffiths A. The nature and measurement of work stress: theory and practice. In: Wilson, JR, Corlett E, Nigel E, et al, eds. Evaluation of human work: a practical ergonomics methodology, 2nd edn. London: Taylor \& Francis, 1995:783-803.

5 Wall TD, Bolden RI, Borrill CS, et al. Minor psychiatric disorder in NHS trust staff: occupational and gender differences. Br J Psychiatry 1997; 171:519-23.

6 University of York. Understanding systematic reviews of research on effectiveness. CDR report 4. York: NHS Centre for Reviews and Dissemination, 1996

7 Agius RM, Blenkin H, Deary IJ, et al. Survey of perceived stress and work demands of consultant doctors. Occup Environ Med 1996:53:217-24.

8 Baglioni Jr AJ, Cooper CL, Hingley P. Job stress, mental health and job satisfaction among UK senior nurses. Stress Medicine 1990;6:9-20.

9 Baldwin PJ, Dodd M, Wrate RM. Young doctors' health-I. How do working conditions affect attitudes, health and performance. Soc Sci Med 1997:45:35-40.

10 Cooper CL, Watts J, Baglioni Jr AJ, et al. Occupational stress amongst general practice dentists. J Occup Psychol 1988;61:163-74

11 Deary IJ, Blenkin H, Agius RM, et al. Models of job-related stress and personal achievement among consultant doctors. Br J Psychol 1996:87:3-29.

12 Heyworth J, Whitley, TS, Allison Jr EJ, et al. Correlates of work-related stress amongst consultants and senior registrars in accident and emergency medicine. Arch Emerg Med 1993;10:279-88.

13 Parkes KR. Occupational stress among student nurses: a natural experiment. J Appl Psychol 1982;67:784-96.

14 Quine L. Workplace bullying in NHS community trust: staff questionnaire study. BM 1999;318:228-32.
15 Rees D, Cooper CL. Occupational stress in health service workers in the UK. Stress Medicine 1992;8:79-90.

16 Sutherland VJ, Cooper CL. Identifying distress among general practitioners: predictors of psychological ill health and job dissatisfaction. Soc Sci Med 1993:37:575-81.

17 Tyler P, Cushway D. Stress, coping and mental well-being in hospital nurses. Stress Medicine 1992;8:91-8.

18 Arsenault A, Dolan SL, Van Ameringen MR. Stress and mental strain in hospital work: exploring the relationship beyond personality. Journal of Organisational Behaviour 1991;12:483-93.

19 Brooke PP, Price JL. The determinants of employees absenteeism: an empirical test of a causal model. J Occup Psychol 1989;62:1-19.

20 Estryn-Behar M, Kaminski J, Peigne E, et al. Stress at work and mental health status among female hospital workers. Br J Ind Med 1990;47:20-8

21 Gray-Toft PA, Anderson JG. Organisational stress in the hospital: development of a model for diagnosis and prediction. Health Serv Res 1985; 19:753-74.

22 Johnson JV, Stewart W, Hall EM, et al. The psychosocial work environment of physicians. J Occup Environ Med 1995;37:1151-9.

23 Landeweerd JA, Boumans NPG. The effect of work dimensions and need for autonomy on nurses' work satisfaction and health. J Occup Organ Psychol 1994;67:207-17.

24 Marshall NL, Barnett RC. Work-related support among women in caregiving occupations. J Community Psychol 1992;20:6-42.

25 Martin TN. Role stress and inability to leave as predictors of mental health. Human Relations 1984:37:969-83.

26 Petterson IL, Arnetz BB, Arnetz JE. Predictors of job satisfaction and job influence: results from a national sample of Swedish nurses. Psychother Psychosom 1995;64:9-19.

27 Pisarski A, Bohle P, Callan VJ. Effects of coping strategies, social support and work-nonwork conflict on shift worker's health. Scand J Work Environ Health 1998;241:41-145.

28 Revicki DA, May HJ. Organisational characteristics, occupational stress, and mental health in nurses. Behav Med 1989;15:30-6.

29 Bacharach SB, Bamberger P, Conley S. Work-home conflict among nurses and engineers: mediating the impact of role stress on burnout and satisfaction at work. Journal of Organisational Behaviour $1991 ; 12: 39-53$.

30 Carayon P, Yang C, Lim S. Examining the relationship between job design and worker strain over time in a sample of office workers. Ergonomics 1995;38:1199-211.

31 Driscoll RJ, Worthington KA, Hurrell Jr JJ. Workplace assault: an emerging job stressor. Consulting Psychology Journal: Practice and Research 1995;47:205-12.

32 Ferrie JE, Shipley M, Marmot MG, et al. An uncertain future: the health effects of threats to employment security in white-collar men and women. Am J Public Health 1998;88:1030-6.

33 Frese $M$. Social support as a moderator of the relationship between work stressors and psychological dysfunctioning: a longitudinal study with objective measures. J Occup Health Psychol 1999:3:179-92.

34 Frone MR, Russell M, Cooper ML. Job stressors, job involvement and employee health: a test of identity theory. J Occup Psychol 1995;68:1-11

35 Fusilier MR, Ganster DC, Mayes BT. Effects of social support, role stress, and locus of control on health. Journal of Management 1987:13:517-28.

36 Karasek Jr RA. Job demands, job decision latitude, and mental strain implications for job redesign. Adm Sci Q 1979;24:285-311.

37 Karasek R. Lower health risk with increased job control among white collar workers. Journal of Organisational Behaviour 1990;11:171-85.

38 LaRocco JM, House JS, French Jr JRP. Social support, occupational stress, and health. J Health Soc Behav 1980;21:202-18.

39 Niedhammer I, Goldberg M, Leclerc A, et al. Psychosocial factors at work and subsequent depressive symptoms in the Gazel cohort. Scand J Work Environ Health 1998:24:197-205.

40 Payne R, Fletcher BC. Job demands, supports, and constraints as predictors of psychological strain among schoolteachers. Journal of Vocational Behaviour 1983;22:136-47.

41 Reifman A, Biernat M, Lang EL. Stress, social support, and health in married professional women with small children. Psychology of Women Quarterly 1991;15:431-45.

42 Romanov K, Appelberg K, Honkasalo M, et al. Recent interpersonal conflict at work and psychiatric morbidity: a prospective study of 15,530 employees aged 24-64. J Psychosom Res 1996;40:169-76.

43 Rubenowitz S, Norrgren F, Tannenbaum AS. Some social psychological effects of direct and indirect participation in ten Swedish companies. Organisation Studies 1983;4:243-59.

44 Shields M. Long working hours and health. Health Reports 1999; 11:33-48.

45 Smulders PGW, Nijhuis, FJN. The job demands-job control model and absence behaviour: results of a 3 -year longitudinal study. Work and Stress 1999;13:115-31

46 Sparks K, Cooper CL. Occupational differences in the work-strain relationship: towards the use of situation-specific models. Journal of Occupational Organizational Psychology 1999;72:219-29.

47 Stansfeld SA, North FM, White I, et al. Work characteristics and psychiatric disorder in civil servants in London. J Epidemiol Community Health 1995;49:48-53.

48 Stansfeld SA, Bosma $\mathrm{H}$, Hemingway $\mathrm{H}$, et al. Psychosocial work characteristics and social support as predictors of SF-36 health functioning: the Whitehall II study. Psychosom Med 1998;60:247-55. 
49 Gronningsaeter H, Hytten K, Skauli G, et al. Improved health and coping by physical exercise or cognitive behavioural stress management training in a work environment. Psychology and Health 1992;7:147-63.

50 Heany CA, Price RH, Refferty J. Increasing coping resources at work: a field experiment to increase social support, improve work team functioning, and enhance employee mental health. Journal of Organisational Behaviour 1995;16:335-52.

51 Kagan NI, Kagan H, Watson MG. Stress reduction in the workplace: the effectiveness of psychoeducational programs. Journal of Counselling Psychology 1995;42:71-8.
52 Lokk J, Arnetz B. Psychophysiological concomitants of organisational change in health care personnel: effects of a controlled intervention study. Psychother Psychosom 1997;66:74-7.

53 Malcolm RM, Harrison J, Forster $\mathrm{H}$. Effects of changing the pattern of referrals in a local authority. Occup Med 1993;43:21 1-15.

54 Smoot SL Gonzales JL. Cost-effective communication skills training for state hospital employees. Psychiatr Serv 1995;46:819-22.

55 Frese $\mathbf{M}$. Stress at work and psychosomatic complaints: a causal interpretation. J Appl Psychol 1985;70:314-28.

$\mathrm{ECHO}$

\section{Air pollution study confirms concerns over childhood rickets}

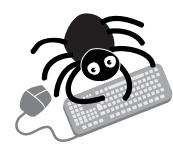

Please visit the Occupational and

Environmental Medicine website [www. occenvmed.com for link to this full article. study in India has shown that young children living in areas of high air pollution are in danger of developing rickets.

Two groups of age matched infants and toddlers were compared for serum vitamin D metabolites, calcium, alkaline phosphatase (AP), and parathormone (PTH) concentrations. One group lived in a central location in Delhi and the other on the outskirts of the city, where air pollution is much lower.

Children from the city centre had significantly lower mean serum total 25-hydroxyvitamin D $(25(\mathrm{OH}) \mathrm{D})$ - an indictor of vitamin D status — than children from the outskirts $(12.4 \mathrm{ng} / \mathrm{ml} v 27.1 \mathrm{ng} / \mathrm{ml})$. Their mean serum AP and PTH concentrations were significantly higher, and the inverse relations between $25(\mathrm{OH}) \mathrm{D}$ and AP, PTH were also significant. Three children had serum total 25(OH)D low enough to indicate rickets, and nine more below adequate amounts. All children from the outskirts had adequate $25(\mathrm{OH}) \mathrm{D}$. Mean haze score was significantly less at the city centre (2.1 against 2.7).

Each group included 34 children aged 9-24 months with similar home conditions, diet, family income, and time spent outside. Blood was taken from 26 children from the city centre and 31 from the outskirts. Haze scores measured at ground level three times daily $(0900,1200,1600)$ during February 2000 were taken as a marker for UVB radiation.

Concerns are growing that increasing air pollution from industry and motor vehicles blocks out UVB radiation and children's ability to make vitamin D naturally, leading to rickets.

A Archives of Disease in Childhood 2002;87:111-113. 